\title{
Enhancing effect of lysine combined with other compounds on cephamycin C production in Streptomyces clavuligerus
}

\author{
Carla A Leite, André P Cavallieri and Maria L G C Araujo*
}

\begin{abstract}
Background: Lysine plays an important role in Streptomyces clavuligerus metabolism; it takes part in its catabolism, via cadaverine, and in its secondary metabolism, in which lysine is converted via 1-piperideine-6-carboxylate to alpha-aminoadipic acid, a beta-lactam antibiotic precursor. The role of lysine as an enhancer of cephamycin C production, when added to production medium at concentrations above $50 \mathrm{mmol} \mathrm{I}^{-1}$, has already been reported in the literature, with some studies attributing a positive influence to multifunctional diamines, among other compounds. However, there is a lack of research on the combined effect of these compounds on antibiotic production.
\end{abstract}

Results: Results from experimental design-based tests were used to conduct response surface-based optimization studies in order to investigate the synergistic effect of combining lysine with cadaverine, putrescine, 1,3-diaminopropane, or alpha-aminoadipic acid on cephamycin C volumetric production. Lysine combined with cadaverine influenced production positively, but only at low lysine concentrations. On the whole, higher putrescine concentrations $\left(0.4 \mathrm{~g} \mathrm{I}^{-1}\right)$ affected negatively cephamycin C volumetric production. In comparison to culture media containing only lysine as additive, combinations of this amino acid with alpha-aminoadipic acid or 1,3-diaminopropane increased cephamycin C production by more than $100 \%$.

Conclusion: This study demonstrated that different combinations of lysine with diamines or lysine with alpha-aminoadipic acid engender significant differences with respect to antibiotic volumetric production, with emphasis on the benefits observed for lysine combined with alpha-aminoadipic acid or 1,3-diaminopropane. This increase is explained by mathematical models and demonstrated by means of bioreactor cultivations. Moreover, it is consistent with the positive influence of these compounds on lysine conversion to alpha-aminoadipic acid, a limiting step in cephamycin C production.

Keywords: Streptomyces clavuligerus, Cephamycin C, Lysine, Diamines, Alpha-aminoadipic acid, Response surface

\section{Background}

The commercial importance of the actinomycete Streptomyces clavuligerus lies in its ability to produce several secondary metabolites of therapeutic interest [1]. Among these compounds are: cephamycin $\mathrm{C}$, a beta-lactam antibiotic more resistant to beta-lactamases than the structurally similar antibiotic cephalosporin $C$ produced by filamentous fungi, and for this reason used as raw material for production of semi-synthetic antibiotics (cefotetan, cefoxitin, cefmetazole, and temocillin) [2,3]; clavulanic

\footnotetext{
* Correspondence: mlaraujo@iq.unesp.br

Department of Biochemistry and Technological Chemistry, UNESP - São

Paulo State University, Institute of Chemistry, 14800-900 Araraquara, SP, Brazil
}

acid, a beta-lactamases inhibitor whose use in conjunction with amoxicillin is the most important commercial example [4]; other clavams, which have antifungal properties [5]; and non-beta-lactam compounds such as holomycin and tunicamycin, which have antibiotic and antitumor properties [5-7]. The biosynthetic diversity inherent to S. clavuligerus results in extremely complex metabolic regulation [8-14], which has led to different studies aimed at increasing the biosynthesis of relevant biocompounds. Among these compounds, cephamycin $\mathrm{C}$ has been one of the most extensively investigated [15-23]. The basic structure of this biocompound and of all other beta-lactam antibiotics produced by prokaryotes or eukaryotes derives from L-cysteine, L-valine, and L-alpha-aminoadipic acid. 
In prokaryotes, alpha-aminoadipic acid is the product of lysine degradation via 1-piperideine-6-carboxylate [24-26]. The use of exogenous lysine to enhance cephamycin $C$ biosynthesis in cultures of producer species has been known for over thirty years [16,20,23,27,28]. Studies have shown that high lysine concentrations (above $50 \mathrm{mmol} \mathrm{l}^{-1}$ ) promote higher cephamycin $\mathrm{C}$ production as compared to that of culture media containing little or no lysine. Researchers have obtained increases of up to $300 \%$ in cephamycin C production by $S$. clavuligerus in a culture medium containing about $100 \mathrm{mmol} \mathrm{l}^{-1}$ of lysine $[14,20,21]$. In spite of lysine degradation via 1-piperideine6-carboxylate pathway producing the precursor alphaaminoadipic acid [25,26], complete lysine catabolism occurs via cadaverine $[24,29,30]$. Cadaverine and other diamines, such as diaminopropane and putrescine, promote beta-lactam antibiotic production in Nocardia lactamdurans or S. clavuligerus [31-34]. Nevertheless, it is difficult to determine the extent to which these compounds influence antibiotic biosynthesis, since diamines act as modulators of several cell functions [32,33,35]. Thus, there is scarce quantitative research on the use of lysine combined with other diamines or other compounds that can potentially enhance beta-lactam antibiotic production in S. clavuligerus [16,23,33]. This was explored in this study, which investigates increases in cephamycin $\mathrm{C}$ production by adding cadaverine, putrescine, 1,3-diaminopropane or alpha-aminoadipic acid in culture media containing lysine as compared to those obtained in culture media containing lysine alone. Cultivations were performed in accordance with a central composite-based, face-centered experimental design (CCF) whereas concentrations of lysine combined with every compound were optimized using Response Surface Methodology. Best conditions were validated by means of batch cultivations in a stirred and aerated benchscale bioreactor.

\section{Methods}

\section{Microorganisms}

Streptomyces clavuligerus ATCC 27064 was stored in the form of spore suspension (approximately $10^{8}$ spores $\mathrm{ml}^{-1}$ ) at $-80^{\circ} \mathrm{C}$ in $2 \mathrm{ml}$ cryotube vials (glycerol at $20 \% \mathrm{w} \mathrm{v}^{-1}$ ).

Escherichia coli ESS 2235 supersensitive to beta-lactam antibiotics was employed as test organism. The strain was cultivated in nutrient agar medium (Difco ${ }^{\mathrm{TM}}$ Nutrient Agar) at $37^{\circ} \mathrm{C}$ for 24 hours. The cells were stored at $-80^{\circ} \mathrm{C}$ in $2 \mathrm{ml}$ cryotube vials.

\section{Culture media}

The seed medium contained $\left(\mathrm{g} \mathrm{l}^{-1}\right)$ tryptone (5.0), yeast extract (3.0), malt extract (10), and buffering agent 3(N-morpholine) propanesulfonic acid (MOPS) (21). The inoculum medium consisted $\left(\mathrm{g} \mathrm{l}^{-1}\right)$ of soluble starch (10), cotton seed extract $\left(\mathrm{PROFLO}^{\oplus}\right.$ - Traders Protein, USA) (8.5), yeast extract (1.0), $\mathrm{K}_{2} \mathrm{HPO}_{4}(0.80), \mathrm{MgSO}_{4} .7 \mathrm{H}_{2} \mathrm{O}$ (0.75), MOPS (21), and $10 \mathrm{ml}$ of salt solution per 1 of medium. The salt solution contained $\left(\mathrm{g} \mathrm{l}^{-1}\right) \mathrm{MnCl}_{2} \cdot 4 \mathrm{H}_{2} \mathrm{O}$ (1.0), $\mathrm{FeSO}_{4} .7 \mathrm{H}_{2} \mathrm{O}$ (1.0), and $\mathrm{ZnSO}_{4} .7 \mathrm{H}_{2} \mathrm{O}$ (1.0). The basal production medium contained $\left(\mathrm{g} \mathrm{l}^{-1}\right)$ soluble starch (10), $\mathrm{PROFLO}^{\circ}$ (8.5) boiled down and filtered (using a vacuum pump), yeast extract (0.50), $\mathrm{K}_{2} \mathrm{HPO}_{4}$ (1.75), $\mathrm{MgSO}_{4} .7 \mathrm{H}_{2} \mathrm{O}$ (0.75), $\mathrm{CaCl}$ (0.20), $\mathrm{NaCl}$ (2.0), MOPS (21), the aforementioned salt solution $\left(5.0 \mathrm{ml} \mathrm{l}^{-1}\right)$, and sodium thiosulfate (1.0) added at $30 \mathrm{~h}$ after inoculation according to Inamine and Birnbaum [31]. The initial $\mathrm{pH}$ of culture media was fitted to $6.8 \pm 0.1$. The proportion of filtered PROFLO ${ }^{\circ}$ nitrogen corresponded to $40 \%$ of gross $\mathrm{PROFLO}^{\circ}$.

In order to investigate the influence of different compounds on cephamycin $\mathrm{C}$ biosynthesis, the production medium was supplemented with lysine, alpha-aminoadipic acid, and diamines 1,3-diaminopropane, putrescine, and cadaverine. These compound concentrations were established according to the purpose of each experiment.

\section{Experimental procedure}

Spore germination and inoculum preparation consisted of two pre-cultures with 24-hour cultivation each in shake flasks. Inoculum volume comprised $10 \%$ of suspension cell volume per culture medium volume throughout this study. Submerged cultures for cephamycin $\mathrm{C}$ production were performed in $500 \mathrm{ml}$ Erlenmeyer shake flasks at $28^{\circ} \mathrm{C}$ and $260 \mathrm{rpm}$ ( $5 \mathrm{~cm}$ eccentricity). To prevent problems of oxygen limitation during the shake-flask procedure, the broth volume was kept under $10 \%$ of the Erlenmeyer flask nominal volume. Samples were collected at 24-hour intervals. Experiments in the bench-scale bioreactor (New Brunswick Bioflo 2000; 51 working volume) were performed at 1.0 vvm aeration rate, $6.8 \pm 0.1 \mathrm{pH}, 28^{\circ} \mathrm{C}$ temperature, and 50\% dissolved oxygen saturation level automatically controlled by varying the agitation speed.

\section{Analytical methods}

The supernatant was obtained after centrifugation of the culture medium at $15,550 \mathrm{x} \mathrm{g}$ for $10 \mathrm{~min}, 4^{\circ} \mathrm{C}$, for further analyses. The cell density was quantified as grams of dry weight per liter of sample (gDWC l-1). Cephamycin $\mathrm{C}$ was determined by means of the agar-diffusion assay method using cephalosporin $C$ zinc salt (Sigma) as standard. Penase $^{\ominus}$ (BD Difco) was employed at $20 \mu \mathrm{L}$ per $\mathrm{ml}$ of sample, reacting at $25^{\circ} \mathrm{C}$ for 20 min to degrade penicillin $\mathrm{N}$. In this method, the measure of cephamycin $C$ represents the total amount of cephalosporins in the sample (in $\mathrm{mg} \mathrm{l}^{-1}$ ) [36]. A calibration curve was performed using ten cephalosporin $\mathrm{C}$ concentration values from 5 to $120 \mathrm{mg} \mathrm{l}^{-1}$ and 24 replicates for each concentration. Antibiotic analyses were also carried out via high-performance liquid chromatography as described in Baptista Neto et al. [37]. Lysine 
Table 1 Range and levels of the independent variables lysine (Lys) and alpha-aminoadipic acid (AAA), in coded and original units, according to the two-factor, three-level central-composite-based, face-centered, experimental design (CCF); the response variable is cephamycin C concentration (CephC) obtained at 72-hour cultivation

\begin{tabular}{|c|c|c|c|c|c|c|}
\hline \multirow[t]{3}{*}{ Run } & \multicolumn{4}{|c|}{ Independent variables } & \multirow{2}{*}{\multicolumn{2}{|c|}{$\begin{array}{c}\text { Response } \\
\text { CephC }\left(\mathrm{mg} \mathrm{l}^{-1}\right)\end{array}$}} \\
\hline & \multicolumn{2}{|c|}{ Coded units } & \multicolumn{2}{|c|}{ Original units $\left(\mathrm{g} \mathrm{l}^{-1}\right)$} & & \\
\hline & $x_{\text {Lys }}$ & $x_{A A A}$ & $x_{\text {Lys }}$ & $x_{A A A}$ & Measured* & Predicted \\
\hline 1 & -1 & -1 & 0.9 & 0 & $25.0 \pm 8.2$ & 15.5 \\
\hline 2 & 0 & -1 & 3.2 & 0 & $45.0 \pm 9.6$ & 52.7 \\
\hline 3 & +1 & -1 & 5.5 & 0 & $55.0 \pm 5.9$ & 56.7 \\
\hline 4 & -1 & 0 & 0.9 & 0.32 & $44.1 \pm 0.9$ & 57.8 \\
\hline 5 & 0 & 0 & 3.2 & 0.32 & $105.8 \pm 6.6$ & 100.5 \\
\hline 6 & +1 & 0 & 5.5 & 0.32 & $118.5 \pm 6.4$ & 110.0 \\
\hline 7 & 0 & +1 & 3.2 & 0.64 & $112.4 \pm 0.0$ & 110.6 \\
\hline 8 & 0 & +1 & 3.2 & 0.64 & $102.8 \pm 0.0$ & 110.6 \\
\hline 9 & 0 & +1 & 3.2 & 0.64 & $117.8 \pm 0.0$ & 110.6 \\
\hline 10 & 0 & +1 & 3.2 & 0.64 & $112.0 \pm 0.0$ & 110.6 \\
\hline 11 & -1 & +1 & 0.9 & 0.64 & $66.7 \pm 7.7$ & 62.4 \\
\hline 12 & +1 & +1 & 5.5 & 0.64 & $118.8 \pm 9.6$ & 125.6 \\
\hline
\end{tabular}

*The cultivations were performed in triplicate, with the exception of cultivation at condition $(0,+1)$ performed in quadruplicate; $S D=$ standard deviation. and alpha-aminoadipic acid analyses were conducted by means of the post-column derivatization method with orthophtalaldehyde and quantified in a fluorescence detector [38]. The starch concentration was determined after acid hydrolysis, by quantifying the total reducing sugars by the dinitrosalicylic acid method [39].

\section{Experimental design}

CCF experimental designs, including four replicates of an experiment under the same conditions, were employed to investigate individual and combined effects of lysine and compounds, one at a time, putrescine, 1,3-diaminopropane, cadaverine, and alpha-aminoadipic acid, on cephamycin $\mathrm{C}$ production. The response surface methodology was used to investigate the relationship between cephamycin $\mathrm{C}$ production (response variable) and the compounds that enhance beta-lactam antibiotic production (independent variables) $[40,41]$. The chosen experimental design and the established concentration limits of the compounds under investigation (independent variables) were appropriate for adjusting models represented by second-order polynomials according to the following equation:

$$
\hat{\mathrm{y}}=\mathrm{a}_{0}+\mathrm{a}_{1} \mathrm{x}_{L y s}+\mathrm{a}_{\mathrm{i}} \mathrm{x}_{i}+\mathrm{a}_{11} \mathrm{x}_{L y s}^{2}+\mathrm{a}_{\mathrm{ii}} \mathrm{x}_{i}^{2}+\mathrm{a}_{1_{\mathrm{i}}} \mathrm{x}_{L y s} \mathrm{x}_{i}
$$

where $\hat{y}$ represents the dependent variable, cephamycin $\mathrm{C}$ production $\left(\mathrm{mg} \mathrm{l}^{-1}\right), \mathrm{a}_{0}$ is the interception coefficient (average value of cephamycin $\mathrm{C}$, in $\mathrm{mg} \mathrm{l}^{-1}$ ), $\mathrm{x}$ represents the independent variables in coded unities: $x_{L y s}=$ lysine, and $x_{i}$ represents the other compounds studied $(\mathrm{i}=$ alpha-

Table 2 Range and levels of independent variables lysine (Lys), 1,3-diaminopropane (1,3D), cadaverine (Cad), and putrescine (Put), in coded and original units, according to two-factor, three-level central-composite-based, face-centered, experimental designs (CCF); the response variable is cephamycin C concentration (CephC) obtained at 72-hour cultivation

\begin{tabular}{|c|c|c|c|c|c|c|c|c|c|c|c|c|}
\hline \multirow[b]{4}{*}{ Run } & \multicolumn{6}{|c|}{ Independent variables } & \multirow{2}{*}{\multicolumn{6}{|c|}{$\begin{array}{c}\text { Response } \\
\text { CephC (mg l-1) }\end{array}$}} \\
\hline & \multirow{2}{*}{\multicolumn{2}{|c|}{$\begin{array}{l}\text { Coded } \\
\text { units }\end{array}$}} & \multirow{2}{*}{\multicolumn{4}{|c|}{ Original units $\left(\mathrm{g} \mathrm{l}^{-1}\right)$}} & & & & & & \\
\hline & & & & & & & \multicolumn{2}{|c|}{ Lys + 1,3D } & \multicolumn{2}{|c|}{ Lys + Cad } & \multicolumn{2}{|c|}{ Lys + Put } \\
\hline & $X_{\text {Lys }}$ & $x_{i}$ & $x_{\text {Lys }}$ & $x_{1,3 D}$ & $X_{C a d}$ & $X_{\text {Put }}$ & Measured* & Predicted & Measured* & Predicted & Measured* & Predicted \\
\hline 1 & -1 & -1 & 0.0 & 0.0 & 0.0 & 0.0 & $18.1 \pm 3.0$ & 10.6 & $19.0 \pm 2.7$ & 22.7 & $18.0 \pm 2.7$ & 16.7 \\
\hline 2 & 0 & -1 & 3.7 & 0.0 & 0.0 & 0.0 & $45.6 \pm 7.2$ & 59.9 & $45.6 \pm 2.2$ & 39.1 & $47.3 \pm 3.2$ & 53.9 \\
\hline 3 & +1 & -1 & 7.4 & 0.0 & 0.0 & 0.0 & $72.3 \pm 4.1$ & 64.9 & $72.1 \pm 1.9$ & 74.7 & $75.5 \pm 3.6$ & 70.3 \\
\hline 4 & -1 & 0 & 0 & 2.5 & 3.5 & 0.2 & $47.6 \pm 3.9$ & 53.9 & $34.7 \pm 3.5$ & 30.2 & $31.1 \pm 2.2$ & 33.8 \\
\hline 5 & 0 & 0 & 3.7 & 2.5 & 3.5 & 0.2 & $108.9 \pm 0.0$ & 109.2 & $40.5 \pm 0.0$ & 41.2 & $63.1 \pm 0.0$ & 64.6 \\
\hline 6 & 0 & 0 & 3.7 & 2.5 & 3.5 & 0.2 & $122.1 \pm 0.0$ & 109.2 & $35.9 \pm 0.0$ & 41.2 & $75.0 \pm 0.0$ & 64.6 \\
\hline 7 & 0 & 0 & 3.7 & 2.5 & 3.5 & 0.2 & $100.7 \pm 0.0$ & 109.2 & $42.0 \pm 0.0$ & 41.2 & $69.0 \pm 0.0$ & 64.6 \\
\hline 8 & 0 & 0 & 3.7 & 2.5 & 3.5 & 0.2 & $120.0 \pm 0.0$ & 109.2 & $41.1 \pm 0.0$ & 41.2 & $64.9 \pm 0.0$ & 64.6 \\
\hline 9 & +1 & 0 & 7.4 & 2.5 & 3.5 & 0.2 & $114.4 \pm 13.6$ & 120.2 & $74.2 \pm 2.1$ & 71.5 & $64.0 \pm 3.4$ & 74.7 \\
\hline 10 & -1 & +1 & 0 & 5.0 & 7.0 & 0.4 & $62.2 \pm 2.6$ & 62.4 & $47.1 \pm 2.8$ & 47.9 & $29.1 \pm 2.5$ & 27.5 \\
\hline 11 & 0 & +1 & 3.7 & 5.0 & 7.0 & 0.4 & $125.3 \pm 0.8$ & 123.7 & $54.1 \pm 0.2$ & 53.5 & $44.9 \pm 2.9$ & 51.9 \\
\hline 12 & +1 & +1 & 7.4 & 5.0 & 7.0 & 0.4 & $140.2 \pm 8.0$ & 140.7 & $78.8 \pm 0.5$ & 78.5 & $61.1 \pm 1.9$ & 55.6 \\
\hline
\end{tabular}

*The cultivations were performed in triplicate, with the exception of cultivation at condition $(0,0)$ performed in quadruplicate; SD $=$ standard deviation. 
aminoadipic, 1,3-diaminopropane, cadaverine or putrescine); $a_{1}$ and $a_{i}$ are the linear terms, $a_{11}$ and $a_{i i}$ are the quadratic terms, $\mathrm{a}_{1 \mathrm{i}}$ is the interaction term. Statistica software (7.0 version) was used for regression and graphical analyses of experimental data.

The conditions of independent variables and cephamycin $\mathrm{C}$ production results (observed and predicted) are shown in Tables 1 and 2.

\section{Results and discussion}

Individual effect of diamines and precursors on

\section{cephamycin C production}

For this study, two concentrations for each diamine were defined based on literature data obtained for other betalactam antibiotic producing microorganisms [32,33,35,42]. Cephamycin $\mathrm{C}$ biosynthesis precursors lysine and alphaaminoadipic acid were tested at several concentrations in order to define ranges of adequate values for the experimental designs.
Cephamycin $\mathrm{C}$ production and cell growth obtained at $48 \mathrm{~h}$ and $72 \mathrm{~h}$ cultivations in basal medium without additives and supplemented with putrescine, 1,3-diaminopropane, and cadaverine are shown in Figure 1. Leitão et al. [32] found that all three diamines promoted cephamycin C production by $N$. lactamdurans, albeit at different levels. The largest increase was observed in culture media containing 2.5 or $5.0 \mathrm{~g} \mathrm{l}^{-1}$ of 1,3-diaminopropane. In this study, this diamine also produced a similar effect: a $100 \%$ increase in volumetric production was observed after the addition of $5.0 \mathrm{~g} \mathrm{l}^{-1}$ of the compound as compared to that of the culture medium with no additive. Also, the addition of 1,3-diaminopropane alone promoted higher specific production than that obtained at the control condition (Figure 1C). Similarly, Martín et al. [42] observed that adding $5.0 \mathrm{mM}\left(0.37 \mathrm{~g} \mathrm{l}^{-1}\right)$ or $10 \mathrm{mM}\left(0.74 \mathrm{~g} \mathrm{l}^{-1}\right)$ of 1,3 diaminopropane enhanced Penicillium chrysogenum betalactam antibiotic production by approximately $100 \%$. It is likely that one of the effects of 1,3-diaminopropane is to
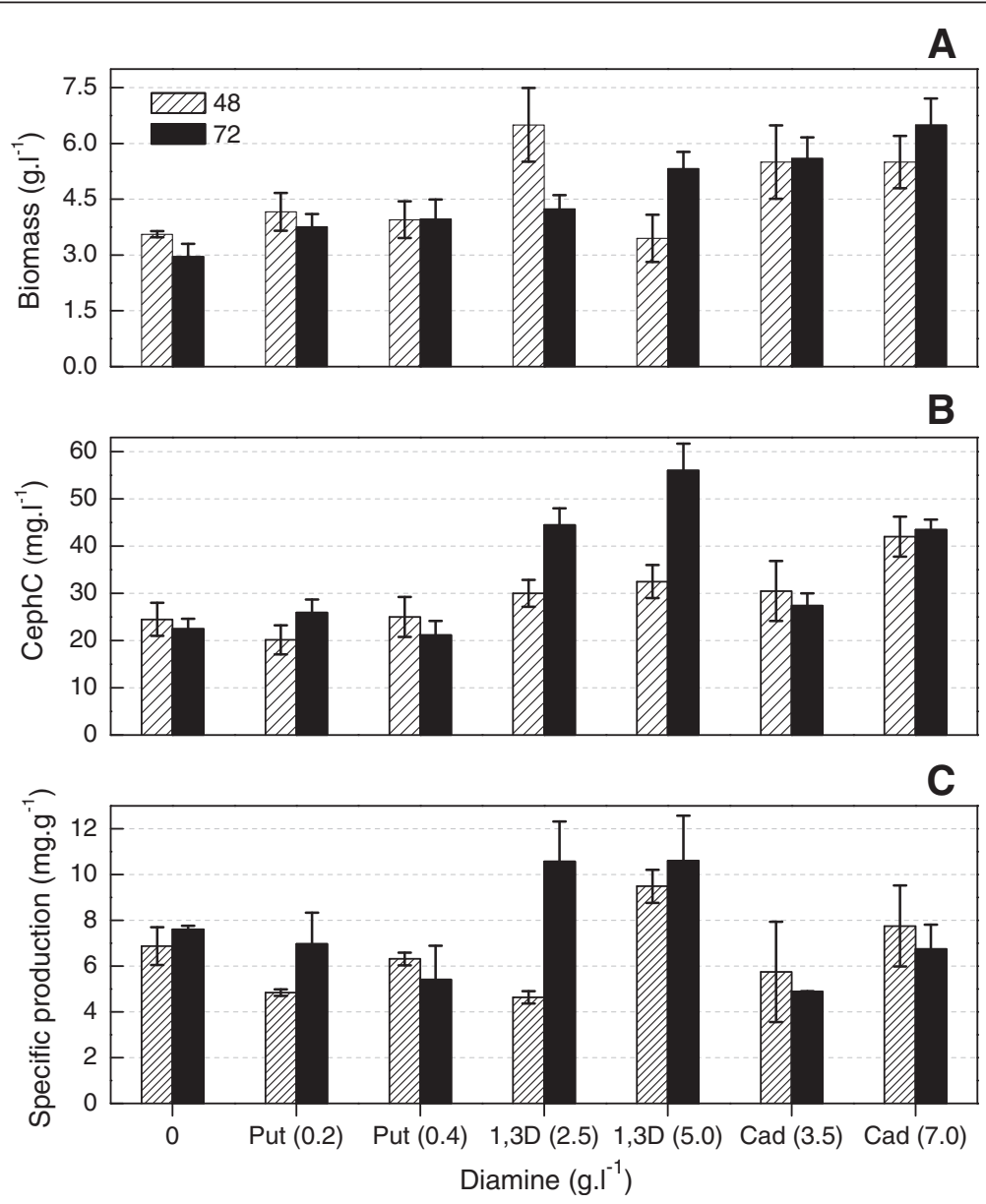

Figure 1 Effect of biomass and cephamycin C with different diamines. Biomass (A), cephamycin C concentration (CephC) (B), and specific production (C) obtained in shake-flasks cultivations of basal medium with no antibiotic-production enhancing compound (control condition) and with putrescine (Put), 1,3-diaminopropane (1,3D), and cadaverine (Cad), at two concentration values (in parentheses); the cultures were performed in triplicate. 
maintain high mRNA transcript levels during the production phase [43].

In the present work, putrescine did not affect antibiotic production by $S$. clavuligerus (Figure 1B), as Martín et al. $[42,43]$ observed with $P$. chrysogenum. However, Leitão et al. [32] observed positive effects on cephamycin C production with $N$. lactamdurans when $0.20 \mathrm{~g} \mathrm{l}^{-1}$ of putrescine was added.

With regard to cadaverine, volumetric production almost doubled by adding $7.0 \mathrm{~g} \mathrm{l}^{-1}$ of this diamine (Figure $1 \mathrm{~B}$ ). However, specific production was not higher than that obtained in media without additives (Figure 1C). For cultivations with $N$. lactamdurans, a threefold increase was obtained using $5.0 \mathrm{~g} \mathrm{l}^{-1}$ of this diamine [32].

In general, the increase in biomass observed at the end of cultivations (Figure 1A) suggests that these diamines acted as sources of carbon and energy $(C)$ and/or nitrogen $(\mathrm{N})$, thereby supplementing the basal medium sources (starch and PROFLO ${ }^{\circ}$ ).

Cephamycin $\mathrm{C}$ production was evaluated at several lysine and alpha-aminoadipic acid concentrations (Figures 2 and 3). Consistent with the literature, high concentrations of exogenous lysine strongly affected cephamycin $\mathrm{C}$ production [20,28]. After adding $14.6 \mathrm{~g} \mathrm{l}^{-1}$ of this amino acid, biomass almost doubled (Figure 2A) and cephamycin $\mathrm{C}$ production increased about six fold (Figure 2B) as compared to data from the basal medium. However, residual concentration values of this amino acid at $14.6 \mathrm{~g} \mathrm{l}^{-1}$ and $18.3 \mathrm{~g} \mathrm{l}^{-1}$ of lysine were approximately $25 \%$ and $35 \%$, respectively. This surplus was not observed at concentrations lower than $11 \mathrm{~g} \mathrm{l}^{-1}$. Moreover, a fivefold global increase in antibiotic volumetric production was obtained between 0 and $11 \mathrm{~g} \mathrm{l}^{-1}$ of lysine, whereas biomass increased only 1.5 times.

Adding up to $1.6 \mathrm{~g} \mathrm{l}^{-1}$ of alpha-aminoadipic acid did not influence biomass formation, which was in the same order of magnitude as that in the basal medium with no additives. Adding $0.64 \mathrm{~g} \mathrm{l}^{-1}$ of alpha-aminoadipic acid to the basal medium resulted in the largest increase in cephamycin $C$ production, four times larger than that obtained with the basal medium. Alpha-aminoadipic acid concentrations higher than $0.64 \mathrm{~g} \mathrm{l}^{-1}$ did not promote higher
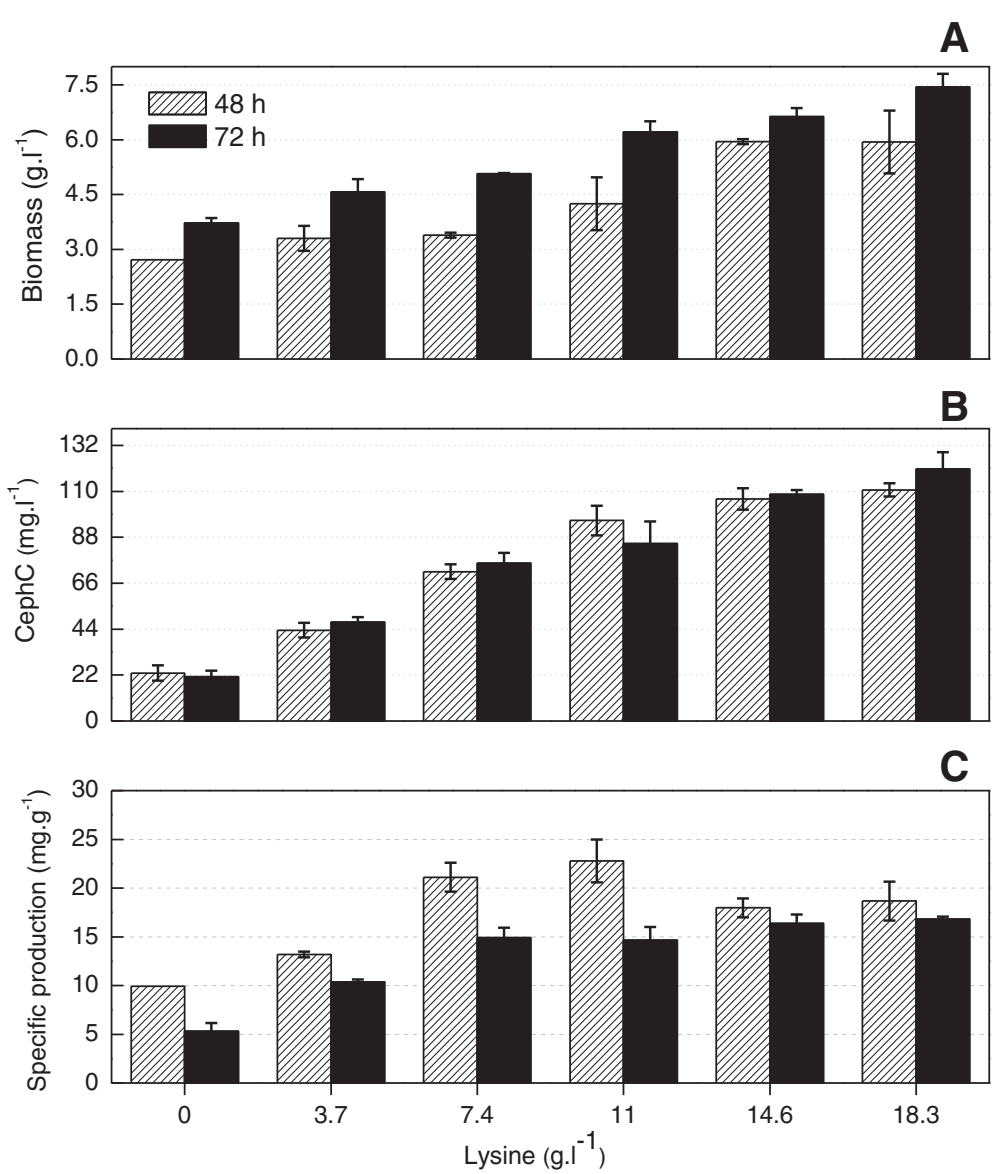

Figure 2 Effect of biomass and cephamycin C with lysine. Biomass (A), cephamycin C concentration (CephC) (B), and specific production (C) obtained from batch cultivations in shaken-flasks of basal medium with no antibiotic-production enhancing compound (control condition) and with lysine (Lys) at different concentration values; the cultures were performed in triplicate. 

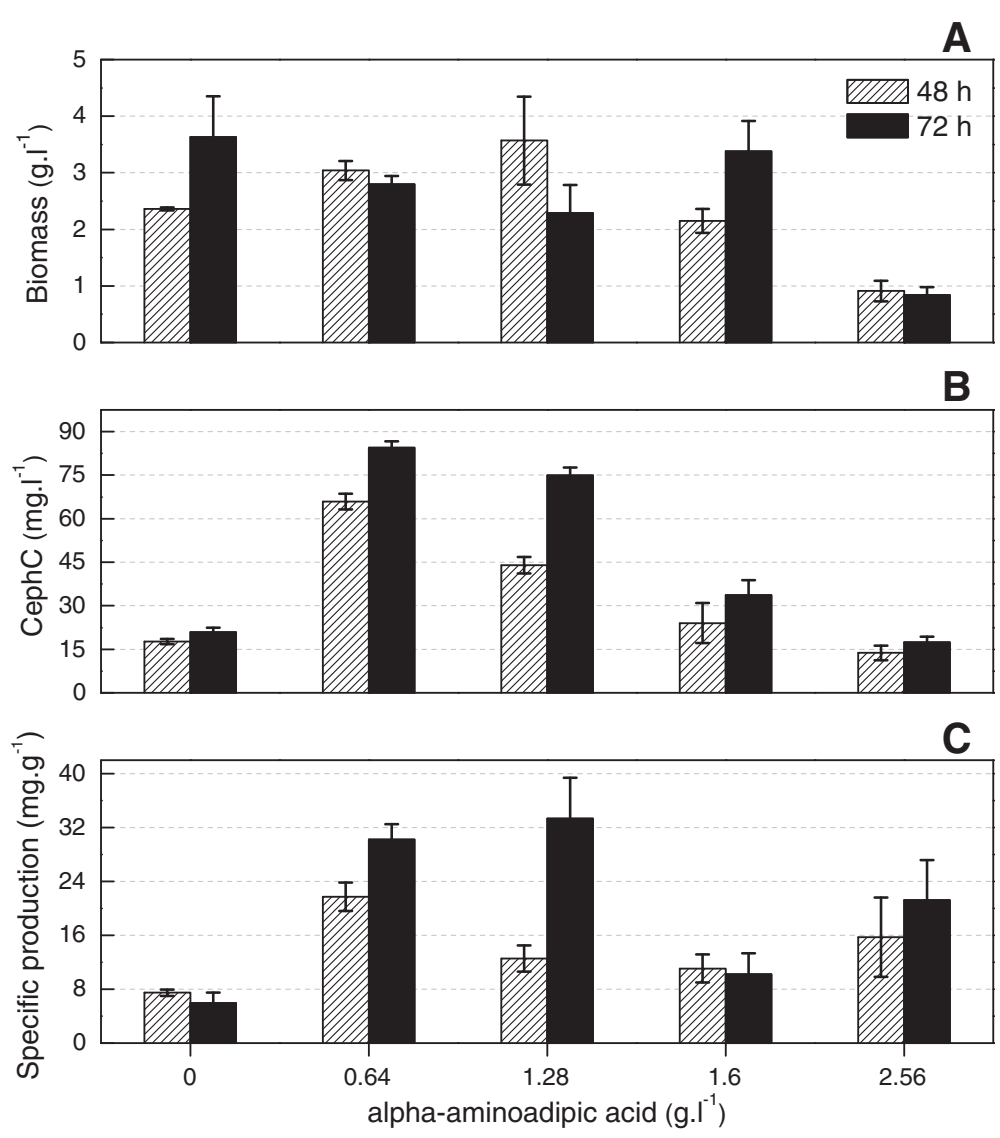

Figure 3 Effect of biomass and cephamycin C with alpha-aminoadipic acid. Biomass (A), cephamycin C concentration (CephC) (B), and specific production (C) obtained from batch cultivations in shaken-flasks of basal medium with no antibiotic-production enhancing compound (control condition) and with alpha-aminoadipic acid (AAA) at different concentration values; the cultures were performed in triplicate.

antibiotic volumetric production, in spite of the amino acid having been completely consumed. Henriksen et al. [44] reported that alpha-aminoadipic acid can be metabolized into 6-oxo-piperideine-2-carboxylic acid (OPC), which is secreted into the culture medium during penicillin production by $P$. chrysogenum. The authors suggested that OPC formation would divert alpha-aminoadipic acid from antibiotic synthesis and lead to lower levels of penicillin production. A similar phenomenon may have occurred in S. clavuligerus.

\section{Effect of lysine in conjunction with diamines or alpha- aminoadipic acid on cephamycin $\mathrm{C}$ production}

The concentration of independent variables enabled the investigation of ranges in which, according to the literature and data from this study, it is possible to maximize cephamycin C production by $S$. clavuligerus or $N$. lactamdurans [16,20,21,31-34,42,43]. Based on results of cultivations using only lysine as additive (Figure 2), concentrations of amino acid ranging from 0 to $7.4 \mathrm{~g} \mathrm{l}^{-1}$ were selected in order to minimize its effect on biomass production. With respect to alpha-aminoadipic acid, concentrations ranging from 0 to $0.64 \mathrm{~g} \mathrm{l}^{-1}$ were selected due to superior cephamycin $\mathrm{C}$ volumetric production results obtained in this range (Figure 3 ).

As to lysine, the highest volumetric production of cephamycin $\mathrm{C}$ was observed at 48 hours, which varied little at 72 hours (Figure 2B). The highest volumetric production values for the basal medium with 1,3-diaminopropane or alpha-aminoadipic acid were observed at 72 hours. With respect to cadaverine and putrescine, the highest volumetric production values observed at 48 and 72 hours were almost the same. For this reason, cultivation time was standardized to 72 hours for the experimental designs and bioreactor processes.

The chosen experimental design (CCF) and the concentration range employed for the compounds under investigation (independent variables), together with the use of response surface methodology for statistical treatment of the data obtained at $72 \mathrm{~h}$ cultivation, allowed for the adjustment of quadratic models to predict cephamycin $\mathrm{C}$ production at $90 \%$ confidence level. The generated response surfaces and their corresponding second-order polynomials are shown in Figure 4. Table 3 shows the 


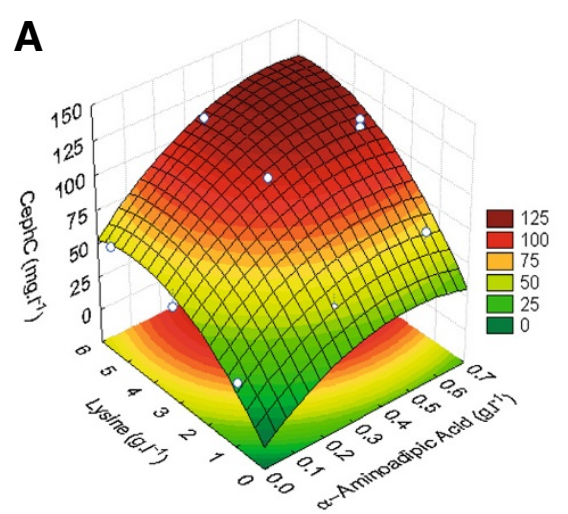

$$
\begin{aligned}
& \hat{y}=76.9( \pm 1.9)+26.1( \pm 2.5) x_{\text {Lys }}+29.0( \pm 2.3) x_{\text {AAA }} \\
& +8.3( \pm 1.9) x_{\text {Lys }}^{2}+9.4( \pm 2.1) x_{\text {AAA }}^{2}
\end{aligned}
$$

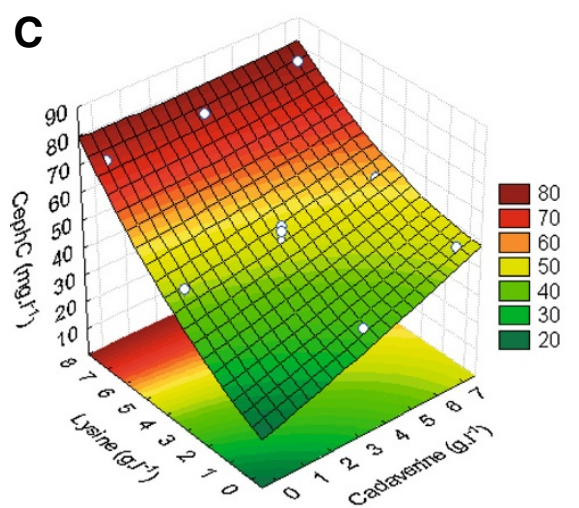

$$
\begin{aligned}
& \hat{y}=52.5( \pm 1.0)+20.7( \pm 1.3) x_{\text {Lys }}-2.6( \pm 1.0) x_{\text {Lys }}^{2} \\
& +7.2( \pm 1.3) x_{\text {Cad }}-5.3( \pm 1.6) x_{\text {Lys }} x_{\text {Cad }}
\end{aligned}
$$

B

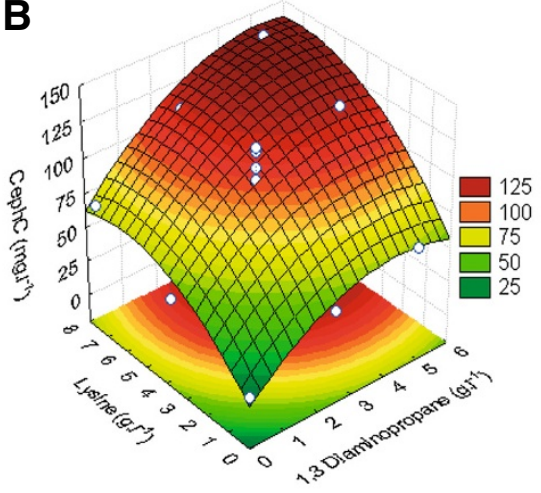

$$
\begin{aligned}
& \hat{y}=82.9( \pm 3.2)+33.2( \pm 4.2) x_{L y s}+31.9( \pm 2.3) x_{1,3 D} \\
& +11.1( \pm 3.2) x_{L y s}^{2}+8.7( \pm 3.2) x_{1,3 D}^{2}
\end{aligned}
$$

D

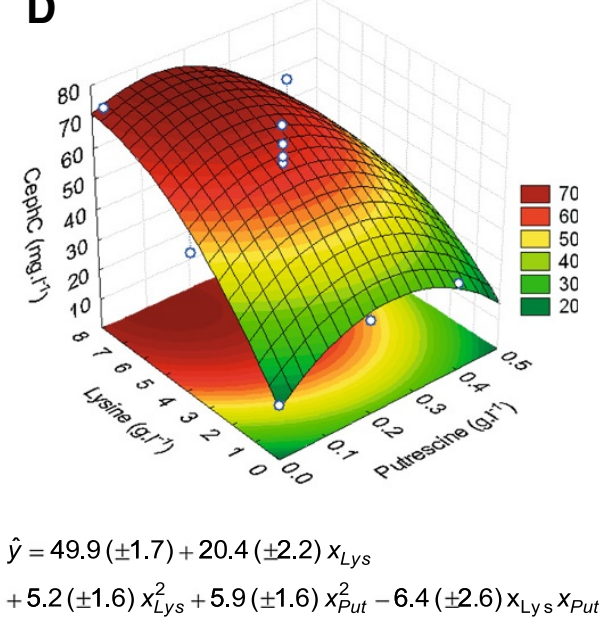

Figure 4 Fitted response surfaces (at $90 \%$ confidence level) for cephamycin C concentration (CephC). Batch cultivation (72-hour) in shaken-flasks in media containing: (A) lysine (Lys) and alpha-aminoadipic acid (AAA), (B) lysine (Lys) and 1,3-diaminopropane (1,3D), (C) lysine (Lys) and cadaverine (Cad), and (D) lysine (Lys) and putrescine (Put).

analyses of variance (ANOVA) of the fitted models, including the F-test to verify the overall significance of each model, its associated probabilities $\mathrm{p}(\mathrm{F})$, and determination coefficient $R^{2}$.

Cephamycin $\mathrm{C}$ production was affected differently for lysine combined with the remaining four compounds. The resulting response surfaces of experimental designs using lysine and alpha-aminoadipic acid (Figure 4A) and lysine and 1,3-diaminopropane (Figure 4B) showed curves and parameters of the same order of magnitude, thereby providing comparable production values. The adjusted mathematical models provide the highest cephamycin $\mathrm{C}$ concentrations of approximately 126 and $140 \mathrm{mg} \mathrm{l}^{-1}$ when $0.6 \mathrm{~g} \mathrm{l}^{-1}$ of alpha-aminoadipic acid and $5.3 \mathrm{~g} \mathrm{l}^{-1}$ of lysine and $5.2 \mathrm{~g} \mathrm{l}^{-1}$ of 1,3-diaminopropane and $7.0 \mathrm{~g} \mathrm{l}^{-1}$ of lysine were added, respectively. In culture media containing just lysine, a production of about $120 \mathrm{mg} \mathrm{l}^{-1}$ was obtained, but only at high amino acid concentrations $\left(14.6 \mathrm{~g} \mathrm{l}^{-1}\right)$ (Figure 2).

It should be remarked that alpha-aminoadipic acid has a strong impact on cephamycin $\mathrm{C}$ production even when added at concentrations nine times lower than those of 1,3-diaminopropane. This is probably due to its being a direct precursor of the beta-lactam antibiotic molecule $[20,21,33]$. On the other hand, 1,3-diaminopropane acts indirectly on beta-lactam antibiotic biosynthesis at the genetic and transcriptional levels [32,43]. Leitão et al. [32] showed that this diamine increases the concentration of lysine-6-aminotransferase and P6C dehydrogenase, which are enzymes responsible for alpha-aminoadipic acid formation. This complex mechanism may support the need for adding larger amounts of 1,3-diaminopropane to produce the same effect as that obtained with alphaaminoadipic acid at lower concentrations, which is in line 
Table 3 Analyses of variance (ANOVA) for the quadratic models regressions at $90 \%$ confidence level

\begin{tabular}{|c|c|c|c|c|c|c|c|c|c|c|}
\hline \multirow[b]{2}{*}{ Source } & \multicolumn{5}{|c|}{ Lysine and alpha-aminoadipic acid $\left(R^{2}=0.9543^{*}\right)$} & \multicolumn{5}{|c|}{ Lysine and 1,3 -diaminopropane $\left(R^{2}=0.9544^{*}\right)$} \\
\hline & SS & DF & MS & $\mathbf{F}$ & $\mathrm{p}$ & SS & DF & MS & $\mathrm{F}$ & $\mathrm{p}$ \\
\hline Model & $13,068.14$ & 5 & $2,613.63$ & $25.06^{* *}$ & $6.0 \times 10^{-4}$ & $15,993.37$ & 5 & 3198.67 & $25.10^{* *}$ & $6.0 \times 10^{-4}$ \\
\hline Residual & 625.82 & 6 & 104.30 & & & 764.58 & 6 & 127.43 & & \\
\hline Lack of fit & 509.35 & 3 & 169.78 & 4.37 & 0.128 & 441.58 & 3 & 147.19 & 1.37 & 0.402 \\
\hline Pure error & 116.47 & 3 & 38.82 & & & 323.00 & 3 & 107.67 & & \\
\hline \multirow[t]{2}{*}{ Total } & $13,693.96$ & 11 & & & & $16,757.95$ & 11 & & & \\
\hline & \multicolumn{5}{|c|}{ Lysine and cadaverine $\left(R^{2}=0.9793^{*}\right)$} & \multicolumn{5}{|c|}{ Lysine and putrescine $\left(R^{2}=0.9006^{*}\right)$} \\
\hline Source & SS & DF & MS & $\mathbf{F}$ & $\mathrm{p}$ & SS & DF & MS & $\mathrm{F}$ & $\mathrm{p}$ \\
\hline Model & $3,080.16$ & 5 & 616.03 & $56.77^{* *}$ & $<10^{-4}$ & $3,650.07$ & 5 & 730.01 & $10.87^{* *}$ & $5.7 \times 10^{-3}$ \\
\hline Residual & 65.10 & 6 & 10.85 & & & 402.82 & 6 & 67.14 & & \\
\hline Lack of fit & 32.35 & 3 & 10.78 & 0.99 & 0.503 & 318.82 & 3 & 106.27 & 3.79 & 0.151 \\
\hline Pure error & 32.75 & 3 & 10.92 & & & 84.00 & 3 & 28.00 & & \\
\hline Total & $3,145.26$ & 11 & & & & 4052.89 & 11 & & & \\
\hline
\end{tabular}

${ }^{*}$ Coefficients of determination for the regressions at $90 \%$ confidence level; SS: Sum of Squares; DF: Degrees of Freedom; MS: Mean Square.

${ }^{* *} \mathrm{~F}_{(5,6)}$ at $90 \%$ confidence level $=3.11$.

with the results obtained in this study. These data and those found in the literature clearly demonstrate, albeit through different methods, that lysine conversion to alpha-aminoadipic acid is a limiting step to cephamycin C biosynthesis. For this reason, adding alpha-aminoadipic acid or 1,3-diaminopropane, though at different concentration levels, was equally effective to overcoming this bottleneck.

Fitted response surfaces for cultivations in culture media containing lysine combined with cadaverine indicate that this diamine only exerts influence on antibiotic production when lysine is added at low concentrations. When the amino acid concentration was increased, the effect of adding diamine gradually waned. It has been suggested that intracellular accumulation of cadaverine may regulate the lysine catabolic pathway through a feedback control mechanism. In this manner, the lysine that would be decarboxylated to form cadaverine is spared, thus increasing lysine supply for cephamycin biosynthesis via the alpha-aminoadipate pathway. The fitted model shows that this behavior only happens at low lysine concentrations. At higher concentrations, lysine would supply both cadaverine and alpha-aminoadipic acid pathways, thereby decreasing the influence of cadaverine. For this reason, as predicted by the model, there is little antibiotic variation (73-77 $\mathrm{mg} \mathrm{l}^{-1}$ of cephamycin C) at the highest lysine concentration $\left(7.4 \mathrm{~g} \mathrm{l}^{-1}\right)$ within the entire cadaverine concentration range under investigation. This is due to the fact that the linear effect of lysine is about thrice stronger than that of this diamine.

With respect to lysine combined with putrescine, adding $0.20 \mathrm{~g} \mathrm{l}^{-1}$ of this diamine to media containing $3.7 \mathrm{~g} \mathrm{l}^{-1}$ of amino acid increased production by approximately $40 \%$ as compared to that obtained with medium containing just lysine at the same concentration (Table 2). On the other hand, adding this diamine to media with higher lysine concentrations $\left(7.4 \mathrm{~g} \mathrm{l}^{-1}\right)$ adversely affected production due to the negative effect stemming from the interaction between the compounds (Figure 4D). Thus, the highest production value predicted for $7.7 \mathrm{~g} \mathrm{l}^{-1}$ of lysine combined with $0.13 \mathrm{~g} \mathrm{l}^{-1}$ of putrescine is just $76 \mathrm{mg} \mathrm{l}^{-1}$. Similar volumetric production values were obtained with basal culture media containing $7.4 \mathrm{~g} \mathrm{l}^{-1}$ of lysine as additive (Figure 2). Martín et al. [43] observed that supplementation with putrescine provided much lower mRNA levels than those obtained with 1,3-diaminopropane in $P$. chrysogenum cultures. Despite structural similarity between 1,3-diaminopropane and putrescine, these authors suggest that the positive effect obtained with diamines is probably attributable to the three-carbon structure of diamines. On the other hand, Leitão et al. [32] observed an approximately threefold increase when $0.2 \mathrm{~g} \mathrm{l}^{-1}$ of putrescine was added to $N$. lactamdurans cultures.

Figures 5 and 6 show the results of two cultivations in bioreactor using $7.0 \mathrm{~g} \mathrm{l}^{-1}$ of lysine combined with $5.2 \mathrm{~g} \mathrm{l}^{-1}$ of 1,3-diaminopropane and $5.3 \mathrm{~g} \mathrm{l}^{-1}$ of lysine combined with $0.64 \mathrm{~g} \mathrm{l}^{-1}$ of alpha-aminoadipic acid. These concentrations, predicted by the models as optimal production conditions, resulted in $190 \mathrm{mg} \mathrm{l}^{-1}$ and $160 \mathrm{mg} \mathrm{l}^{-1}$ of cephamycin $\mathrm{C}$ for lysine combined with 1,3-diaminopropane and lysine combined with alpha-aminoadipic acid, respectively.

When compared to top values predicted by the mathematical models, these results represent increases of approximately $35 \%$ for lysine combined with 1,3diaminopropane and approximately $27 \%$ for lysine combined with alpha-aminoadipic acid. While diamine supplementation favored cell growth, because it can 


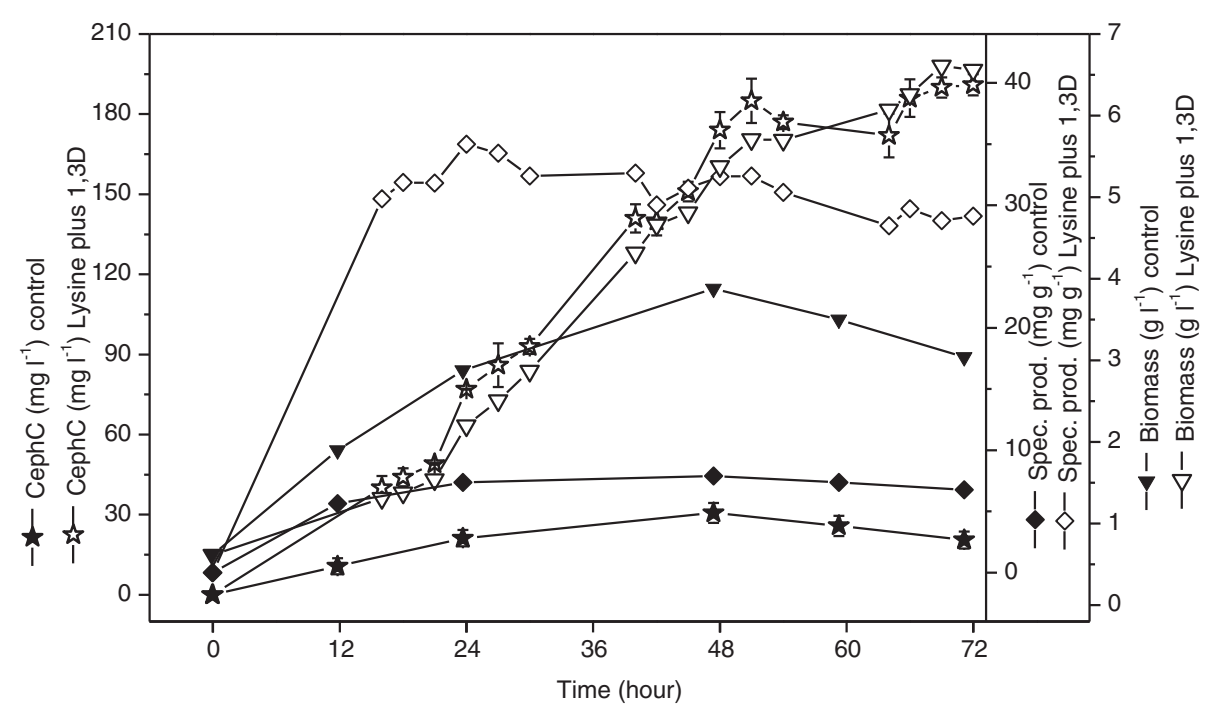

Figure 5 Batch cultivation in agitated and aerated bench-bioreactor for lysine combined with 1,3-diaminopropane. Cephamycin C concentration (CephC), specific production, and biomass; basal medium containing cephamycin C production-enhancing compounds at their optimal values (in parentheses), lysine $\left(7.0 \mathrm{~g} \mathrm{l}^{-1}\right)$ and 1,3-diaminopropane $\left(5.2 \mathrm{~g} \mathrm{l}^{-1}\right)$ (open symbols); control condition: basal medium without additives (solid symbols).

act as an additional source of $\mathrm{C}$ and $\mathrm{N}$, alpha-aminoadipic acid did not affect biomass production. Thus, the specific production at the end of cultivation with lysine combined with alpha-aminoadipic acid was approximately $30 \%$ higher as compared to that of lysine combined with 1,3-diaminopropane, reaching values of up to $40 \mathrm{mg} \mathrm{l}^{-1}$ and $30 \mathrm{mg} \mathrm{l}^{-1}$, respectively. Results obtained in bioreactor employing medium without additives (control condition) are also shown in Figures 5 and 6.

\section{Conclusions}

It has been known for a long time that adding lysine enhances cephamycin $C$ production. However, its use as the sole enhancer does not take full advantage of the antibiotic productivity of $S$. clavuligerus. In this study, an experimental design method (CCF) and Response Surface Methodology are successfully employed to adjust mathematical models to describe the effects of lysine combined with cadaverine, putrescine, 1,3-diaminopropane or alpha-aminoadipic acid on cephamycin $\mathrm{C}$ production

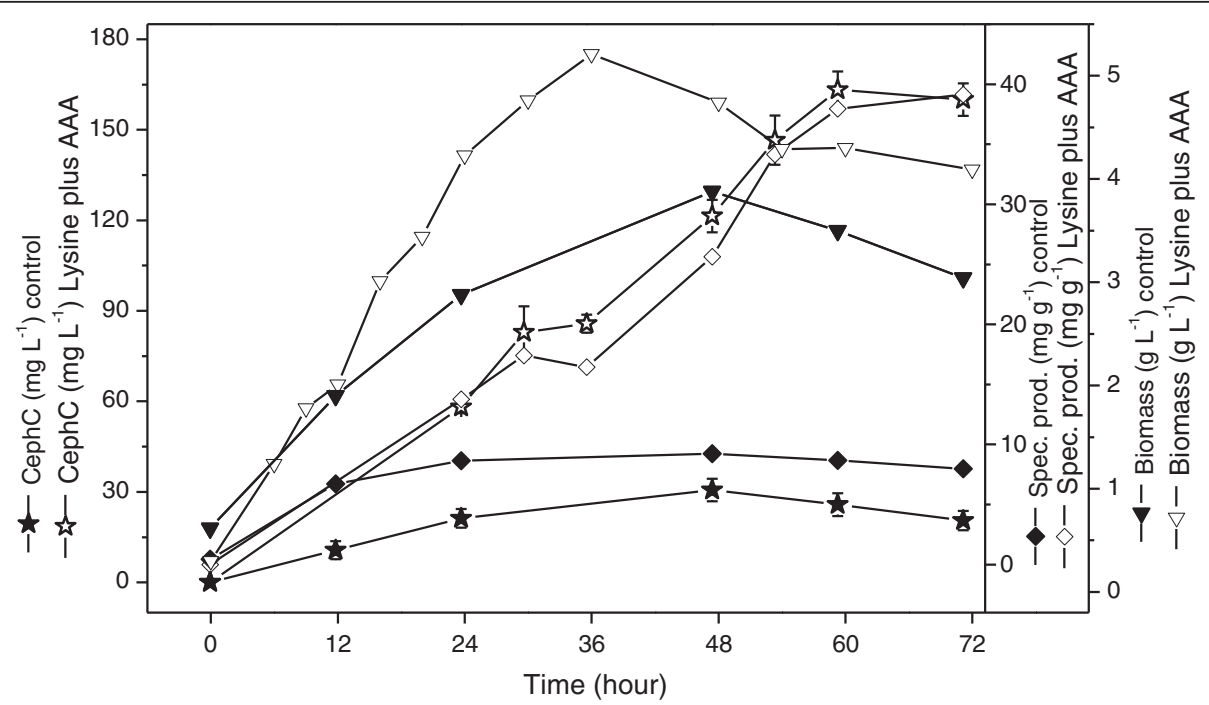

Figure 6 Batch cultivation in agitated and aerated bench-bioreactor for lysine combined with alpha-aminoadipic acid. Cephamycin C concentration (CephC), specific production, and biomass; basal medium containing cephamycin C production-enhancing compounds at their optimal values (in parentheses), lysine $\left(5.3 \mathrm{~g}^{\mathrm{I}^{-1}}\right.$ ) and alpha-aminoadipic acid $\left(0.6 \mathrm{~g} . \mathrm{I}^{-1}\right)$ (open symbols); control condition: basal medium without additives (solid symbols). 
by $S$. clavuligerus. Moreover, the interactions observed and validated by the fitted models are shown to be compatible to biochemical data already established in the literature about the pathway of beta-lactam antibiotics in S. clavuligerus. This study demonstrates that different combinations of lysine with other compounds promote significant variations in antibiotic production, with emphasis on the benefits obtained from using lysine combined with alpha-aminoadipic acid or 1,3-diaminopropane. These combinations increased cephamycin $\mathrm{C}$ production by more than $100 \%$ as compared to that with culture media containing just lysine as additive at the same concentrations. This positive effect may be attributed to alpha-aminoadipic acid or 1,3-diaminopropane in conjunction with lysine acting to overcome the bottleneck caused by lysine conversion to alpha-aminoadipic acid, albeit via different mechanisms. In the case of lysine combined with cadaverine, there was a positive effect on cephamycin $\mathrm{C}$ production by the diamine, especially when lysine was added at low concentrations. Cadaverine acted by decreasing lysine catabolism. However, as the amino acid concentration increased, the diamine effect waned, as the model clearly indicates. On the other hand, the highest volumetric production obtained with lysine combined with putrescine was approximately twice lower than that obtained with lysine combined with alpha-aminoadipic acid or 1,3diaminopropane.

\section{Competing interests}

All the authors of the submitted work (CA, AP, and MLGC) declare that there has been no financial relationship or support from any company in the past five years. We declare too that there are no competing interests, whether political, personal, religious, ideological, academic, intellectual or commercial, or any other activities influencing the submitted work.

\section{Authors' contributions}

CA carried out the assays with the diamines (experimental designs and fermentation in bioreactor), and was responsible for the agar bioassays and handling, storage, and maintenance of the microorganisms (Streptomyces clavuligerus ATCC 27064 and Escherichia coli ESS 2235). AP carried out the assays with alpha-aminoadipic acid (experimental designs and fermentation in bioreactor), and was responsible for the analyses in high-performance liquid chromatography (amino acids, C and N sources, antibiotics). MLGC designed and coordinated the study and performed its statistical analysis. All authors collaborated on the text, interpreting and discussing the results, and approved the final manuscript.

\section{Authors' information}

CA - biologist; PhD student in Biotechnology, Institute of Chemistry, Araraquara campus, Department of Biochemical and Technological Chemistry, Laboratory of Bioprocesses; AP - biochemist and pharmacist, PhD student in Biotechnology, Institute of Chemistry, Araraquara campus, Department of Biochemical and Technological Chemistry, Laboratory of Bioprocesses; MLGC - chemical engineer and PhD in Chemical Engineering and expertise in industrial microbiology and fermentation for almost 25 years, assistant Professor at UNESP (São Paulo State University) Institute of Chemistry, Araraquara campus, Department of Biochemical and Technological Chemistry, Laboratory of Bioprocesses.

\section{Acknowledgements}

The authors gratefully acknowledge the financial support grant 2005/55079-4; 2008/52819-5 and 2013/02632-4, São Paulo Research Foundation (FAPESP) and Dr. Paloma Liras (Facultad de Ciencias Biológicas y Ambientales,
Universidad de León, León, Spain) for kindly donating E. coli ESS 2235, a test organism supersensitive to beta-lactam antibiotics.

Received: 12 August 2013 Accepted: 18 December 2013 Published: 20 December 2013

\section{References}

1. Challis GL, Hopwood DA: Synergy and contingency as driving forces for the evolution of multiple secondary metabolite production by Streptomyces species. Proc Natl Acad Sci USA 2003, 100:14555-14561.

2. Omstead DR, Hunt GH, Buckland BC: Commercial production of cephamycin antibiotics. In Comprehensive biotechnology. Edited by Moo-Young M. New Jersey: Pergamon Press; 1985:187-210.

3. Goldstein EJC, Citron DM: Annual incidence, epidemiology, and comparative in vitro susceptibilities to cefoxitin, cefotetan, cefmetazole, and ceftizoxime of recent community-acquired isolates of the Bacteroides fragilis. J Clin Microbiol 1988, 26:2361-2366.

4. Domingues LCG, Teodoro JC, Hokka CO, Badino AC, Araujo MLGC: Optimisation of the glycerol-to-ornithine molar ratio in the feed medium for the continuous production of clavulanic acid by Streptomyces clavuligerus. Biochem Eng J 2010, 53:7-11.

5. de la Fuente A, Lorenzana LM, Martín JF, Liras P: Mutants of Streptomyces clavuligerus with disruptions in different genes for clavulanic acid biosynthesis produce large amounts of holomycin: possible crossregulation of two unrelated secondary metabolic pathways. J Bacteriol 2002, 184:6559-6565.

6. Kenig M, Reading C: Holomycin and an antibiotic (MM 19290) related to tunicamycin, metabolites of Streptomyces clavuligerus. J Antibiot 1979, 32:549-554.

7. Price NPJ, Tsvetanova B: Biosynthesis of the tunicamycins: a review. J Antibiot 2007, 60:485-491.

8. Khetan A, Malmberg LH, Kyung YS, Sherman DH, Hu WS: Precursor and cofactor as a check valve for cephamycin biosynthesis in Streptomyces clavuligerus. Biotechnol Prog 1999, 15:1020-1027.

9. Tahlan K, Anders C, Jensen SE: The paralogous pairs of genes involved in clavulanic acid and clavam metabolite biosynthesis are differently regulated in Streptomyces clavuligerus. J Bacteriol 2004, 186:6286-6297.

10. Jensen SE, Wong A, Griffin A, Barton B: Streptomyces clavuligerus has a second copy of the proclavaminate amidinohydrolase gene. Antimicrob Agents Chemother 2004, 48:514-520.

11. Liras P, Martín JF: Gene clusters for beta-lactam antibiotics and control of their expression: why have clusters evolved, and from where did they originate? Int Microbiol 2006, 9:9-19.

12. Gomez-Escribano JP, Martín JF, Hesketh A, Bibb MJ, Liras P: Streptomyces clavuligerus relA-null mutants overproduce clavulanic acid and cephamycin C: negative regulation of secondary metabolism by ( $p$ ) ppGpp. Microbiol 2008, 154:744-755.

13. Yin $H$, Xiang $S$, Zheng J, Fan $K$, Yu T, Yang $X$, Peng $Y$, Wang H, Feng D: Induction of holomycin production and complex metabolic changes by the $\operatorname{argR}$ mutation in Streptomyces clavuligerus NP1. Appl Environ Microbiol 2012, 78:3431-3441.

14. Ozcengiz G, Demain AL: Recent advances in the biosynthesis of penicillins, cephalosporins and clavams and its regulation. Biotechnol Adv 2013, 31:287-311.

15. Aharonowitz Y, Demain AL: Carbon catabolite regulation of cephalosporin production in Streptomyces clavuligerus. Antimicrob Agents Chemother 1978, 14:159-164.

16. Mendelovitz S, Aharonowitz Y: Regulation of cephamycin C synthesis, aspartokinase, dihydrodipicolinic acid synthetase, and homoserine dehydrogenase by aspartic acid family amino acids in Streptomyces clavuligerus. Antimicrob Agents Chemother 1982, 21:74-84.

17. Lebrihi A, Lefebvre G, Germain P: A study on the regulation of cephamycin $C$ and expandase biosynthesis by Streptomyces clavuligerus in continuous and batch culture. Appl Microbiol Biotechnol 1988, 28:39-43.

18. Okabe M, Kuwajima T, Satow M, Kimura K, Okamura K, Okamoto R: Preferential and high-yield production of a cephamycin $\mathrm{C}$ by dissolved oxygen controlled fermentation. J Ferment Bioeng 1992, 73:292-296.

19. Malmberg LH, Hu WS, Sherman DH: Efects of enhanced lysine $\varepsilon$-aminotransferase activity on cephamycin biosyntesis in Streptomyces clavuligerus. App/ Microbiol Biotechnol 1995, 44:198-205. 
20. Fang A, Keables $P$, Demain AL: Unexpected enhancement of beta-lactam antibiotic formation in Streptomyces clavuligerus by very high concentrations of exogenous lysine. Appl Microbiol Biotechnol 1996, 44:705-709.

21. Rius N, Maeda K, Demain AL: Induction of L-lysine $\varepsilon$-aminotransferase by $\mathrm{L}$-lysine in Streptomyces clavuligerus, producer of cephalosporins. FEMS Microbiol Lett 1996, 144:207-211.

22. Kota KP, Sridhar P: Solid state cultivation of Streptomyces clavuligerus for cephamycin C production. Process Biochem 1999, 34:325-328.

23. Bussari B, Saudagar PS, Shaligram NS, Survase SA, Singhal RS: Production of cephamycin C by Streptomyces clavuligerus NT4 using solid-state fermentation. J Ind Microbiol Biotechnol 2008, 35:49-58.

24. Kern BA, Hendlin D, Inamine E: L-lysine eps-aminotransferase involved in cephamycin C synthesis in Streptomyces lactamdurans. Antimicrob Agents Chemother 1989, 17:679-685.

25. de la Fuente JL, Rumbero A, Martín JF, Liras P: Delta-1-Piperideine-6carboxylate dehydrogenase, a new enzyme that forms alphaaminoadipate in Streptomyces clavuligerus and other cephamycin C-producing actinomycetes. J Biochem 1997, 327:59-64.

26. Pérez-Llarena FJ, Rodríguez-García A, Enguita FJ, Martín JF, Liras P: The pcd gene encoding piperideine-6-carboxylate dehydrogenase involved in biosynthesis of alpha-aminoadipic acid is located in the cephamycin cluster of Streptomyces clavuligerus. J Bacteriol 1998, 180:4753-4756.

27. Mendelovitz S, Aharonowitz Y: Beta-lactam antibiotic production by Streptomyces clavuligerus mutants impaired in regulation of aspartokinase. J Gen Microbiol 1983, 129:2063-2069.

28. Leitão AL, Enguita FJ, Martín JF, Oliveira JFS: Effect of exogenous lysine on the expression of early cephamycin C biosynthetic genes and antibiotic production in Nocardia lactamdurans MA4213. Appl Microbiol Biotechnol 2001, 56:670-675.

29. Madduri K, Stuttard C, Vining LC: Lysine catabolism in Streptomyces spp. is primarily through cadaverine: beta-lactam producers also make alpha-aminoadipate. J Bacterio/ 1989, 171:299-302.

30. Madduri K, Shapiro S, DeMarco AC, White RL, Stuttard C, Vining LC: Lysine catabolism and alpha-aminoadipate synthesis in Streptomyces clavuligerus. Appl Microbiol Biotechnol 1991, 35:358-363.

31. Inamine E, Birnbaum J: Fermentation of cephamycin C. US Patent 1976, 3:977,942.

32. Leitão AL, Enguita FJ, Fuente $J L$, Liras $P$, Martín JF: Inducing effect of diamines on transcription of the cephamycin c genes from the lat and pcbab promoters in Nocardia lactamdurans. J Bacteriol 1999, 181:2379-2384

33. Demain AL, Vaishnav P: Involvement of nitrogen-containing compounds in $\beta$-lactam biosynthesis and its control. Crit Rev Biotechnol 2006, 26:67-82.

34. Kagliwal LD, Survase SA, Singhal RS: A novel medium for the production of cephamycin $C$ by Nocardia lactamdurans using solid-state fermentation. Bioresour Technol 2009, 100:2600-2606.

35. Igarashi K, Kashiwagi K: Modulation of cellular function by polyamines. Int J Biochem Cell Biol 2010, 42:39-51.

36. Liras P, Martín JF: Assay methods for detection and quantification of antimicrobial metabolites produced by Streptomyces clavuligerus: microbial processes and products. In Methods in Biotechnology. Volume 18: Microbial processes and Products. Edited by Barredo JL. New Jersey: Humana Press; 2005:149-163

37. de Baptista Neto Á, Bustamante MCC, Oliveira JHHL, Granato AC, Bellão C, Junior ACB, Barboza M, Hokka CO: Preliminary studies for cephamyin C purification technique. App/ Biochem Biotechnol 2012, 166:208-221.

38. Dorresteijn RC, Berwald LG, Zomer G, De Gooijer CD, Wieten G, Beuvery EC: Determination of amino acids using o-phthalaldehyde-2-mercaptoethanol derivatization - effect of reaction conditions. J Chromatogr A 1996, 724:159-167.

39. Miller GL: Use of dinitrosalicylic acid reagent for determination of reducing sugar. Anal Chem 1959, 31:426-428.

40. Box GEP, Hunter JS, Hunter WG: Statistics for experimenters: design, innovation, and discovery. 2nd edition. New York: John Wiley and Sons; 2005

41. Rodrigues MI, lemma AF: Planejamento de experimentos e otimização de processos. Casa do Pão Editora: Campinas SP; 2005.
42. Martín J, Estrada CG, Rumbero A, Recio E, Albillos SM, Ullán RV, Martín JF: Characterization of an autoinducer of penicillin biosynthesis in Penicillium chrysogenum. Appl Environ Microb 2011, 77:5688-5696.

43. Martín J, Estrada CG, Kosalková K, Ullán RV, Albillos SM, Martín JF: The inducers 1,3-diaminopropane and spermidine produce a drastic increase in the expression of the penicillin biosynthetic genes for prolonged time, mediated by the LaeA regulator. Fungal Genet Biol 2012, 49:1004-1013.

44. Henriksen CM, Nielsen J, Villadsen J: Cyclization of alpha-aminoadipic acid into the delta-lactam 6-oxo-piperidine-2-carboxylic acid by Penicillium chrysogenum. J Antibiot 1998, 51:99-106.

doi:10.1186/1471-2180-13-296

Cite this article as: Leite et al:: Enhancing effect of lysine combined with other compounds on cephamycin C production in Streptomyces clavuligerus. BMC Microbiology 2013 13:296.

\section{Submit your next manuscript to BioMed Central and take full advantage of:}

- Convenient online submission

- Thorough peer review

- No space constraints or color figure charges

- Immediate publication on acceptance

- Inclusion in PubMed, CAS, Scopus and Google Scholar

- Research which is freely available for redistribution 